\title{
Effectiveness of Self-Help Plus in Preventing Mental Disorders in Refugees and Asylum Seekers in Western Europe: A Multinational Randomized Controlled Trial
}

\author{
Marianna Purgato ${ }^{a, b}$ Kenneth Carswell ${ }^{c}$ Federico Tedeschi ${ }^{a} \quad$ Ceren Acarturk $^{d}$ Minna Anttila ${ }^{e}$ \\ Teresa Au $^{c}$ Malek Bajbouj ${ }^{f} \quad$ Josef Baumgartnerg ${ }^{g}$ Massimo Biondi ${ }^{h}$ Rachel Churchill ${ }^{i}$ \\ Pim Cuijpers ${ }^{j, m}$ Markus Koesters ${ }^{k}$ Chiara Gastaldon ${ }^{a, b}$ Zeynep Ilkkursun ${ }^{d}$ Tella Lantta ${ }^{e}$ \\ Michela Nosè ${ }^{a, b} \quad$ Giovanni Ostuzzi ${ }^{a}$ b Davide Papola a,b Mariana Popal Valentina Roselli ${ }^{\text {h }}$ \\ Marit Sijbrandijj,m Lorenzo Tarsitani ${ }^{\text {h }}$ Giulia Turrini ${ }^{a}$ b Maritta Välimäki $^{\mathrm{e}}$ Lauren Walker $^{\mathrm{n}}$ \\ Johannes Wancatag Elisa Zanini ${ }^{\mathrm{a}}$ Ross White ${ }^{\mathrm{l}}$ Mark van Ommeren ${ }^{\mathrm{c}}$ Corrado Barbui ${ }^{\mathrm{a}, \mathrm{b}}$ \\ ${ }^{a}$ WHO Collaborating Centre for Research and Training in Mental Health and Service Evaluation, Department of \\ Neuroscience, Biomedicine and Movement Sciences, University of Verona, Verona, Italy; ${ }^{\mathrm{b}}$ Cochrane Global Mental \\ Health, University of Verona, Verona, Italy; ${ }^{C}$ Department of Mental Health and Substance Use, World Health \\ Organization, Geneva, Switzerland; ' Department of Psychology, College of Social Sciences and Humanities, Koc \\ University, Istanbul, Turkey; ${ }^{e}$ Department of Nursing Science, University of Turku, Turku, Finland; ${ }^{f}$ Department of \\ Psychiatry, Campus Benjamin Franklin, Charité, Universitätsmedizin, Berlin, Germany; ${ }^{9}$ Clinical Division of Social \\ Psychiatry, Department of Psychiatry and Psychotherapy, Medical University of Vienna, Vienna, Austria; ${ }^{\mathrm{h}}$ Department \\ of Human Neurosciences, Sapienza University of Rome, Rome, Italy; 'Centre for Review and Dissemination, University of \\ York, York, UK; ' Department of Clinical, Neuro, and Developmental Psychology, Amsterdam Public Health Institute, Vrije \\ Universiteit Amsterdam, Amsterdam, The Netherlands; ${ }^{k}$ Department of Psychiatry II, Ulm University, Ulm, Germany; \\ 'Institute of Population Health, University of Liverpool, Liverpool, UK; ${ }^{\mathrm{m}}$ WHO Collaborating Centre for Research and \\ Dissemination of Psychological Interventions, Vrije Universiteit Amsterdam, Amsterdam, The Netherlands; ${ }^{\mathrm{n}}$ Mental \\ Health and Addiction Research Group, University of York, York, UK
}

\section{Keywords}

Randomized controlled trial · Psychological stress ·

Psychological well-being · Psychological trauma - Adult .

Refugees · Asylum seekers · Self-Help Plus

\section{Abstract \\ Introduction: Self-Help Plus ( $\mathrm{SH}+$ ) is a group-based psycho- logical intervention developed by the World Health Organi- zation for managing stress. Objective: To assess the effec- tiveness of $\mathrm{SH}+$ in preventing mental disorders in refugees and asylum seekers in Western Europe. Methods: We con-}

ducted a randomized controlled trial in 5 European countries. Refugees and asylum seekers with psychological distress (General Health Questionnaire score $\geq 3$ ), but without a Diagnostic and Statistical Manual of Mental Disorders, 5th edition (DSM-5) or ICD/10 diagnosis of mental disorder, as assessed with the Mini International Neuropsychiatric Interview (MINI), were randomized to $\mathrm{SH}+$ or enhanced treatment as usual (ETAU). The primary outcome was the frequency of mental disorders with the MINI at 6 months. Sec-

M. von Ommeren and C. Barbui contributed equally to this study. karger@karger.com www.karger.com/pps

Karger $\stackrel{\text { ' }}{5}$ BOPEN ACCESS
(C) 2021 The Author(s)

Published by S. Karger AG, Basel

This is an Open Access article licensed under the Creative Commons Attribution-NonCommercial-4.0 International License (CC BY-NC) (http://www.karger.com/Services/OpenAccessLicense), applicable to the online version of the article only. Usage and distribution for commercial purposes requires written permission.
Correspondence to:

Marianna Purgato, marianna.purgato@univr.it 
ondary outcomes included the frequency of mental disorders at postintervention, self-identified problems, psychological symptoms, and other outcomes. Results: Four hundred fiftynine individuals were randomly assigned to $\mathrm{SH}+$ or ETAU. For the primary outcome, we found no difference in the frequency of mental disorders at 6 months (Cramer $V=0.007, p=$ $0.90, \mathrm{RR}=0.96 ; 95 \% \mathrm{Cl} 0.52-1.78$ ), while the difference significantly favored $\mathrm{SH}+$ at after the intervention (secondary outcome, measured within 2 weeks from the last session; Cramer $V=0.13, p=0.01, \mathrm{RR}=0.50 ; 95 \% \mathrm{Cl} 0.29-0.87)$. Conclusions: This is the first randomized indicated prevention study with the aim of preventing the onset of mental disorders in asylum seekers and refugees in Western Europe. As a prevention effect of $\mathrm{SH}+$ was not observed at 6 months, but rather after the intervention only, modalities to maintain its beneficial effect in the long term need to be identified.

(C) 2021 The Author(s)

Published by S. Karger AG, Basel

\section{Introduction}

Refugees and asylum seekers are at an elevated risk of developing psychological symptoms and mental disorders in association with the numerous challenges that they experience before, during, and after migration [1-7]. In response to such challenges, a growing body of research has demonstrated the effectiveness of mental health interventions in treating psychological symptoms experienced by refugees and asylum seekers [8-16]. However, in relation to prevention, a recent Cochrane systematic review identified only 7 randomized controlled trials (RCT) examining the preventive effects of psychosocial interventions in humanitarian settings [15]. Of these, many did not systematically exclude participants with a probable mental disorder at baseline, and most did not assess for the development of a probable mental disorder at follow-up. Indeed, the review found no studies that evaluated incidence, and no overall preventive effects were identified for other outcomes such as symptom reduction or functioning [15].

The World Health Organization (WHO) has developed a novel, low-intensity, 5-session, group-delivered, guided self-help intervention for managing stress and coping with adversity. It is designed to be delivered by nonspecialist facilitators and does not require extensive training [17]. This psychological intervention, called SelfHelp Plus $(\mathrm{SH}+)$, is intended to help people cope with distress stemming from diverse types of adversity and to enhance psychological flexibility [17]. SH+ was recently tested in a large cluster RCT with the aim of reducing psy- chological distress and improving functioning at a 3-month follow-up in a group of 694 severely distressed South Sudanese female refugees in Uganda [18]. In comparison with enhanced treatment as usual (ETAU), $\mathrm{SH}+$ led to a significant reduction in psychological distress immediately after the intervention $(\beta=-3.25 ; 95 \% \mathrm{CI}-4.31$ to $-2.19, p<0.0001, \mathrm{~d}=-0.72$ ) and at the 3-month followup $(ß=-1.20 ; 95 \% \mathrm{CI}-2.33$ to $-0.08, p=0.04, \mathrm{~d}=-0.26)$.

The easily implementable structure of $\mathrm{SH}+$, its contents, and its delivery modality may make this intervention a potentially useful public health strategy for preventing mental disorders in refugees and asylum seekers living in high-income countries. We therefore evaluated $\mathrm{SH}+$ as an indicated preventive intervention for reducing the frequency of current mental disorders at a 6-month follow-up in refugees and asylum seekers experiencing psychological distress, but without mental disorders, as compared with ETAU. We additionally tested $\mathrm{SH}+$ on a broader range of secondary outcomes, including psychological symptoms, functioning, wellbeing, perceived psychological problems, quality of life, and postmigration living difficulties.

\section{Materials and Methods}

Study Design

We conducted a rater-blind, parallel group, multinational RCT in 5 countries (Italy, Germany, Austria, Finland, and 2 sites in the UK, i.e., 1 in England and 1 in Scotland). The trial protocol was registered in clinicaltrials.gov (NCT03571347) before the start of recruitment and submitted and published in a scientific journal after the start of recruitment [19]. No changes were made to the study design after the start of the trial. An individual level randomization was chosen because of potential heterogeneity across clusters located in different European countries.

Participant recruitment occurred from September 1, 2018, to November 30, 2019. Project coordinators at each site approached local organizations providing social, health, and/or legal support to refugees and asylum seekers to identify potentially eligible participants. Based on a situational analysis of international migration flows, we identified refugees and asylum seekers from Syria, Afghanistan, Pakistan, Iraq, and Nigeria as potential target groups. All screening, baseline, and follow-up assessment questionnaires were administered in interview format or self-administered after the participants signed informed consent forms. Assessors were healthcare professionals who were trained in the administration of rating scales, instructed on how to perform follow-up assessments while preserving effective masking, and assisted by cultural mediators when needed.

\section{Randomization and Masking}

Randomization was centralized and coordinated by the WHO Collaborating Centre of the University of Verona. The randomization schedule was generated by the electronic software Castor Elec- 
tronic Data Capture (EDC) [20], employing a variable block randomization method (block sizes: 4 and 6). Research team members involved in recruitment were able to access the web-based software to randomize each newly enrolled participant, but they were not able to access the randomization list and they were not aware of the block size. Castor EDC software allowed random allocation only after the main information on the enrolled participant had been entered, upon verification of the inclusion criteria. After random allocation, the software produced a unique identification number (ID) for each participant.

Masking of participants and research staff was not feasible due to the nature of the intervention. However, assessors evaluating primary and secondary outcomes, and the statistician performing the analyses, were masked to the participant allocation status. The trial statistician was not involved in determining the participants' eligibility, in administering the intervention, in measuring the outcomes, or in entering data.

\section{Inclusion and Exclusion Criteria}

Refugees and asylum seekers at 1 of the 6 sites of the 5 European countries who presented psychological distress as assessed by the 12-item General Health Questionnaire (GHQ $\geq 3$ ) [21] but did not meet the criteria for a mental disorder according to the Mini International Neuropsychiatric Interview (MINI) [22] were eligible to be randomly assigned to $\mathrm{SH}+$ or ETAU in a 1:1 ratio. The MINI was designed as a brief structured interview for the diagnosis of current mental disorders in the Diagnostic and Statistical Manual of Mental Disorders, 5th edition (DSM-5) [23] and the ICD-10 classification of mental and behavioral disorders [24]. Participants were excluded if they: (1) had a mental disorder according to the MINI [22]; (2) had an acute medical condition contraindicating study participation; (3) presented clinical evidence of an imminent suicide risk or a suicide risk scored as "moderate or high" (or a suicidality disorder according to the MINI suicidality module); (4) were not able to understand Dari, Urdu, Arabic, or English; or (5) showed signs of impaired decision-making as evidenced by responses during the clinical interview. Refugees and asylum seekers who were excluded because of a diagnosis of a mental disorder and/or an imminent suicide risk were referred to professional treatment.

\section{Experimental and Control Intervention}

The intervention delivery period lasted from September 2018 until March 2020. The SH+ program was developed by the WHO, as described elsewhere [17]. $\mathrm{SH}+$ consists of a prerecorded audio course delivered by briefly trained facilitators in a group setting and complemented with an illustrated self-help book adapted for the cultural groups included in the study. It was recently updated and published by the WHO as Doing What Matters in Times of Stress: An Illustrated Guide [25].

The prerecorded audio format of $\mathrm{SH}+$ is innovative in that it seeks to ensure that key intervention components are delivered as intended without the burden of extensive facilitator training. The $\mathrm{SH}+$ program is based on acceptance and commitment therapy (ACT), a form of cognitive-behavioral therapy [26]. ACT emphasizes learning new ways to accommodate difficult thoughts and feelings while guiding people to take proactive steps toward living in a way that is consistent with their values [17].

$\mathrm{The} \mathrm{SH}+$ prerecorded audio material is delivered across five 2-h sessions to groups of up to 30 people. The audio material im- parts key information about stress management and guides participants through individual exercises and small-group discussions. To augment the audio recordings, an illustrated self-help book reviews all essential content and concepts.

The SH+ intervention was fully delivered in the native language of the participants by trained facilitators with a migration background, who were native speakers of the target languages and proficient in English. Most facilitators had no prior work experience in this field and/or formal mental health training. Facilitators completed 5 days of training, which included listening to the audio recordings, receiving instruction on $\mathrm{SH}+$ facilitation skills, and role-playing and practicing $\mathrm{SH}+$ sessions. The facilitator's role consisted of playing the audio, responding to questions and disruptions, demonstrating the exercises, and reading out scripted discussion questions.

Intervention supervision was provided by clinical psychologists or other health care professionals, who were also available for questions, discussion, and debriefing after the sessions. If necessary, additional training and consultations were available from $\mathrm{SH}+$ expert trainers at the WHO through local visits. Fidelity was checked by the intervention supervisor through session adherence forms completed by the facilitators. In addition, the intervention supervisor (a clinical psychologist, a psychiatrist, or a specialized nurse with a formal background in mental health) observed at least $10 \%$ of the sessions and completed an adherence form for each $\mathrm{SH}+$ session.

ETAU was provided to the control group and consisted of routinely delivered social support and/or care according to local regulations. Additionally, participants in the ETAU arm received baseline and follow-up assessments according to the study schedule (around 2 and 6 months after randomization, respectively), information about freely available health and social services, and links to community networks providing support to refugees and asylum seekers.

An ethics advisory board, consisting of experts giving advice on any ethical issues related to the trial, supervised this study.

\section{Outcomes}

The primary outcome was the presence of current mental disorders at the 6-month follow-up measured using the MINI, which was also administered as a screener, and after the intervention [22]. Validation and reliability studies have been conducted comparing the MINI to the Structured Clinical Interview for DSM-III-R [27] and the Composite International Diagnostic Interview, i.e., a structured interview developed by the WHO [28].

All other measures were secondary outcomes assessed at after the intervention and at the 6-month follow-up. Psychological distress was measured using the GHQ-12 questionnaire, asking whether the respondent has experienced a particular symptom or behavior recently [21]. Each item is rated on a 4-point Likert scale and gives a total score of 12 points based on the GHQ version and on the selected scoring methods. PTSD symptoms were assessed with the PTSD Checklist for DSM-5 (PCL-5) [29, 30], a 20-item questionnaire that measures PTSD symptom severity globally (score $0-80$ ) and by cluster (intrusions, avoidance, negative changes in thoughts and mood, and changes in arousal). We measured depression symptoms with the Patient Health Questionnaire, 9-item version (PHQ-9) [31], a 4-point scale measure (score 0 to 27). Personally identified problems were examined with the Psychological Outcome Profiles instrument (PSYCHLOPS) [32], ask- 
ing participants to describe 2 problems from their own perspective and rate the problem severity on a 6-point scale (range $0-18$ ). Functional impairment and subjective wellbeing were assessed with the WHO Disability Assessment Schedule 2.0 (WHODAS) [33] and the WHO-5 Wellbeing Index (WHO-5) [34], respectively. We administered the 12-item version of the WHODAS, which uses a 5 -point scale (score 12-60). The WHO-5 contains 5 questions using a 6-point scale (score 0-25). For evaluation of general health, we administered the EQ-5D-3L questionnaire, which is a brief selfreport measure consisting of 5 dimensions (mobility, self-care, usual activities, pain/discomfort, and anxiety/depression) [35]. Traumatic/adverse life events and daily and environmental stressors were collected with the Harvard Trauma Questionnaire (HTQ)Part A [36] and the 17-item Checklist for Post-Migration Living Difficulties (PMLD) [37]. HTQ-Part A asks for lifetime traumatic life events. The PMLD instead asks respondents to rate their experience of the problems, during the last 12 months, on a 5-point scale (from "was not a problem" to "very serious problem").

Assessments were conducted as face-to-face interviews or remotely by telephone or secure online audio/video communication for reasons of feasibility related to the SARS-CoV2 pandemic. Adverse events reported spontaneously by the participants or observed by the research staff were recorded, reviewed by the ethics advisory board in regular meetings, and reported to the WHO Ethics Committee in midterm reports.

Researchers involved in screening, baseline, and follow-up assessments received specific 2 - $\mathrm{h}$ training sessions for administering the MINI and the rating scales for measuring secondary outcomes by expert trainers based at the University of Verona.

In addition, we assessed secondary outcomes at the 12-month follow-up, as well as cost-effectiveness indicators. The results from these assessments will be presented elsewhere.

\section{Statistical Analysis}

We predicted a frequency rate of current mental disorders of $25 \%$ at 6 months in this population. We hypothesized that the provision of $\mathrm{SH}+$ would show a clinically significant advantage by producing a between-groups absolute difference of $10 \%$ [19]. With these figures, in order to achieve at least $80 \%$ power for a 0.05 level of significance in a $\chi^{2}$ test for equality of proportions of people diagnosed with current mental disorders at 6 months, a sample size of 500 participants (250 in each group) was needed.

Descriptive statistics (mean and SD for continuous variables and absolute numbers and percentages for categorical variables) were calculated on sociodemographic, premigration, migration, and postmigration variables at baseline. Balance between treatment groups was checked by calculating standardized mean differences (SMD) using values of 0.1 and -0.1 as thresholds for imbalance $[38,39]$.

We followed an intention-to-treat (ITT) approach for the analysis of primary and secondary outcomes. The ITT population consisted of all participants randomly assigned to the competing intervention strategies, and with data on the baseline assessment available, irrespectively of the number of $\mathrm{SH}+$ sessions received. To check the robustness of results, the primary outcome was additionally analyzed using a per protocol (PP) approach for confirmatory purposes only, by including only those participants who completed at least $3 \mathrm{SH}+$ sessions.

The primary outcome was compared between the 2 groups through a $\chi^{2}$ test (primary analysis); the Cramer $V$ was also calcu- lated, together with a RR and its 95\% CI (Cramer $V$ details are in the online suppl. Appendix; for all online suppl. material, see www. karger.com/doi/10.1159/000517504). Additionally, a multivariate analysis (secondary analysis) was performed through a Poisson regression model, with a robust error variance, to explore the potential confounding effect of prognostic factors, and the interactions with treatment, controlling for variables unbalanced at baseline.

We also performed a mixed analysis of covariance (ANCOVA) controlling for baseline scores, with robust standard errors and distinct variances for postintervention and the 6-month follow-up. In addition to mixed models, a last observation carried forward (LOCF) approach was used to account for missing observations at 6 months. Standardized coefficients, together with their SEs, were also calculated with the Stata "stdBeta" command [40]. We tested the null hypothesis of the intervention having no effect on any outcomes versus the alternative hypothesis of the intervention having an effect on at least 1 outcome by performing a seemingly unrelated regression (SUR) [41] equations model, in its modification to allow for unbalanced data as proposed by Baum and Schaffer [42], through the Stata "suregub" command. In particular, SURs were performed for each time point, controlling for baseline values. For each questionnaire, in case of missing items, we used the corrected item mean substitution method (i.e., the item mean across participants weighted by the subject's mean of completed items) [43], using information from subjects belonging to the same treatment arm for the same follow-up time, through the Stata "hotvalue" command [44]. The substitution was only performed for observations having $<50 \%$ missing items and if it resulted in admissible values in all cases. As a sensitivity analysis, we reran our models without any data imputation.

Possible interactions between treatment and specific variables (recruiting center, country of origin, gender, age, years of education, and length of stay in the hosting country) were evaluated. In particular, in the case of continuous outcomes, SURs for unbalanced data on all outcomes was performed, with their value at baseline, treatment status, all potential moderators, and their interactions with treatment status as predictors. A global test on all interaction terms was implemented and, in case of statistical significance, the same test was performed for each scale. Finally, for scales meeting the statistical significance threshold, single regressions were considered.

As for binary outcomes, to avoid the issue of poor performance of the model in case of solutions near the boundary described in Zhu et al. [45], Poisson regression models with robust standard errors, having the variable "intervention allocation," each variable separately, and its interaction with treatment as regressors, were performed using the Bonferroni correction to take multiple testing into account. Originally, further analyses stratifying by method of assessment (face-to-face vs. telephone or secure online audio/video communication) were planned. However, due to the SARSCoV2 pandemic, these analyses were not performed, as most follow-up assessments were conducted remotely.

For each secondary outcome, we performed multivariate analyses to take confounding factors into account, again including the baseline value as a covariate. Finally, lost-to-follow-up was compared between the 2 groups using a $\chi^{2}$ or a Fisher exact test, as appropriate. We also conducted 2 post hoc analyses, i.e., 1 analysis using the LOCF approach for the MINI at the 6-month follow-up by imputing missing values using information after the intervention and 1 analysis considering deterioration of psychological 


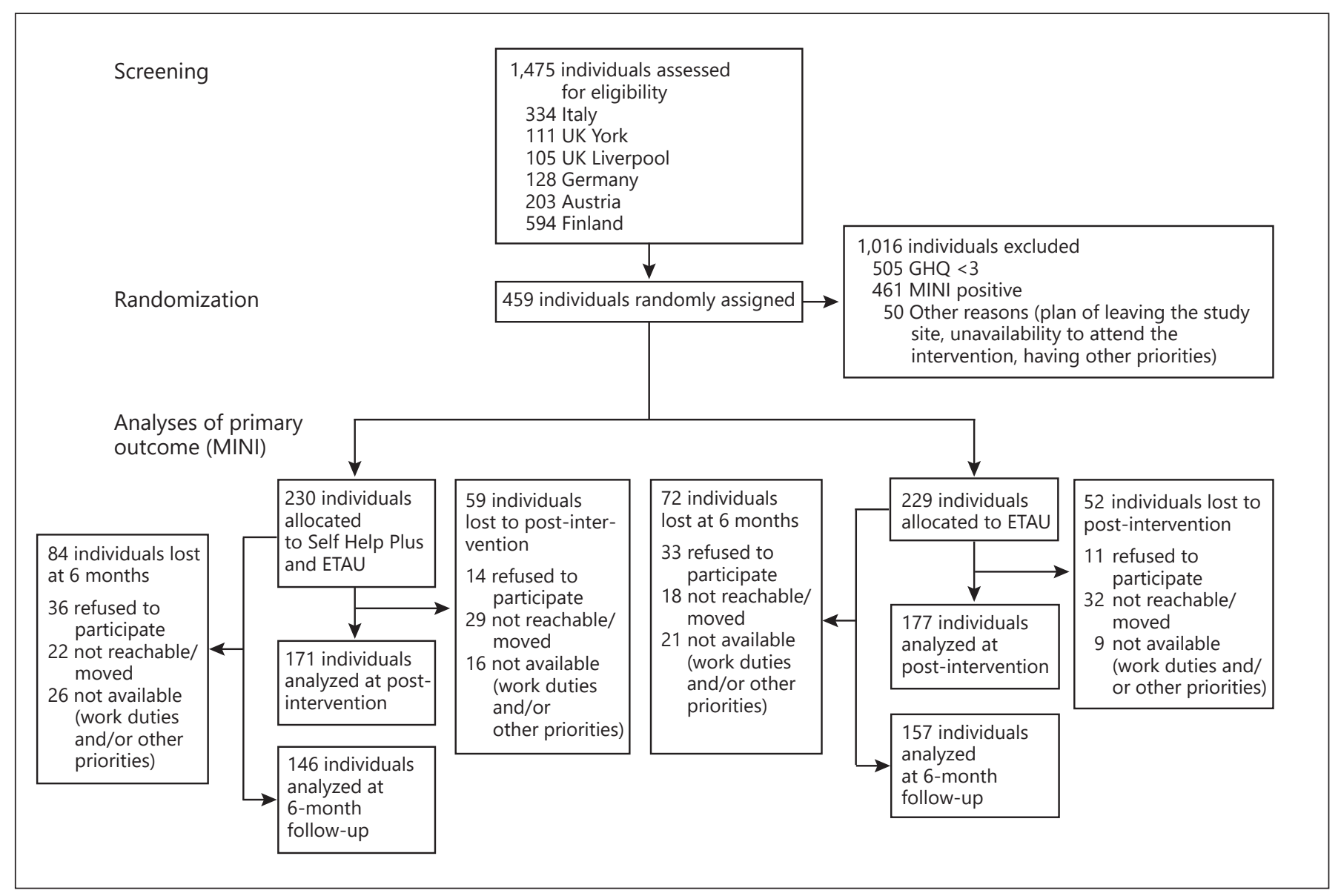

Fig. 1. CONSORT flow diagram.

symptoms (PHQ-9, PCL-5, and WHODAS) after the intervention. All analyses were performed using Stata/SE, release 16.1 [46]. A statistical analysis plan was developed and signed before data analysis.

\section{Results}

After screening of 1,475 potentially eligible participants, 1,016 were excluded. A total of 505 participants were excluded because the level of distress was lower than the established cut-off, 461 participants were excluded because of a positive MINI, and 50 participants were excluded for other reasons (Fig. 1).

This left 459 individuals who met the inclusion criteria, consented to be randomized by signing a written informed consent form, and were allocated to either $\mathrm{SH}+$ (230) or ETAU (229). We could not assess 112 individuals at post-intervention with the M.I.N.I. (24\%), of which
59/230 in SH+ group and 53/229 in ETAU group, and 156 individuals at 6-month follow-up (34\%), of which $84 / 230$ in $\mathrm{SH}+$ group and 72/229 in ETAU. Participants were lost to 6-month follow-up because they refused to participate (69), were not reachable and/or moved to other locations (40), or were not available due to other personal priorities (i.e., working, housing, other) (47). The distribution of participants lost to follow-up was similar between the study groups (Table 1).

Selected sociodemographic characteristics of the included participants are shown in Table 2 (the full list is presented in the online suppl. Appendix, Table 1).

The mean participant age was 33 years $(S D=11)$ in the $\mathrm{SH}+$ group and 32 years $(\mathrm{SD}=10)$ in the ETAU group. Approximately 7 out of every 10 participants were male. For about $40 \%$ primary school was the highest level of education, while $20 \%$ had received academic education, with 10 years of education on average. About $28 \%$ of the participants came from Syria, 25\% 
Table 1. Summary statistics of the results for primary and secondary outcomes at each time point

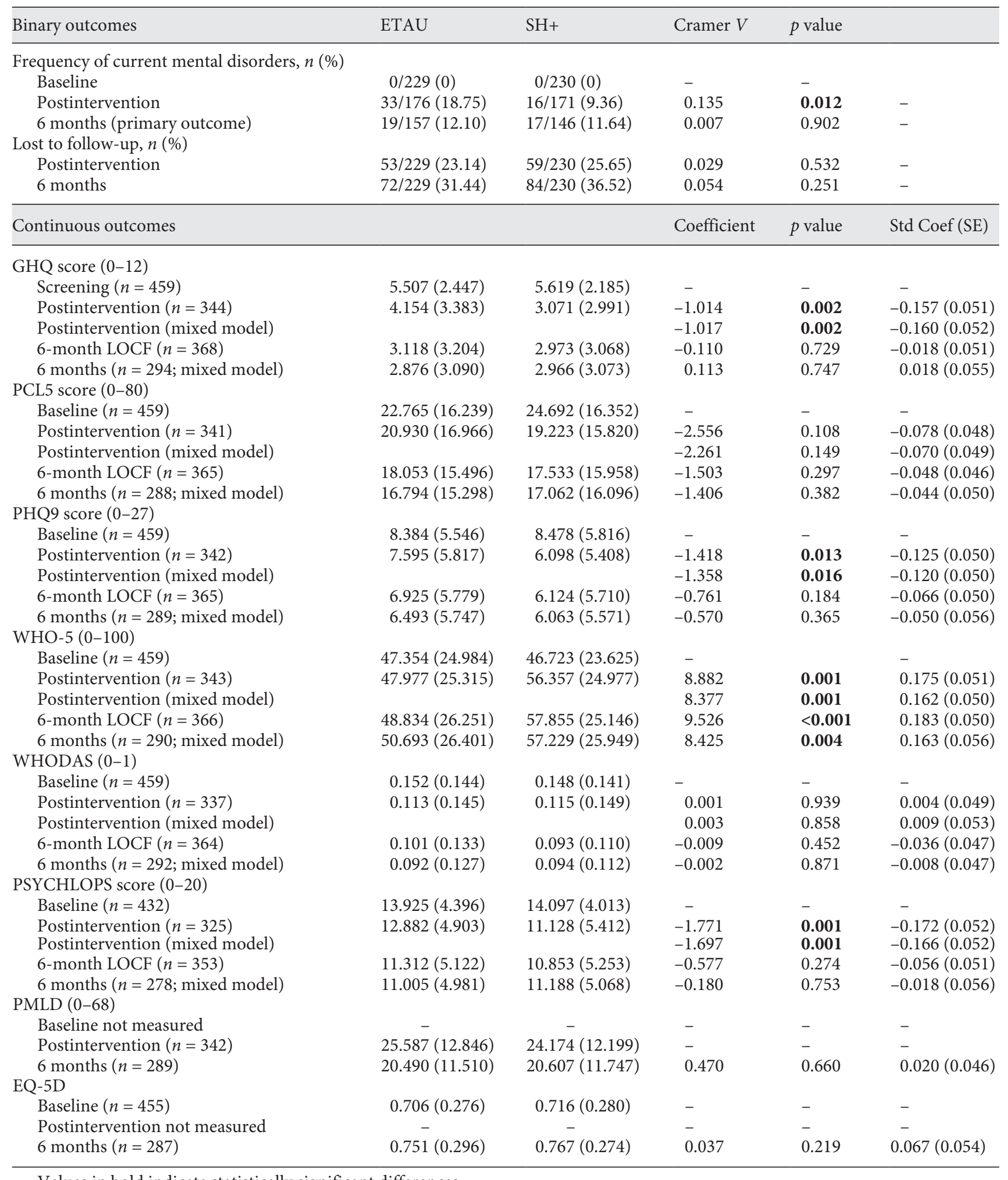

Values in bold indicate statistically significant differences. 
Table 2. Demographic characteristics

\begin{tabular}{|c|c|c|c|c|}
\hline Variable & ETAU & $\mathrm{SH}+$ & Difference (SE) & SMD \\
\hline Mean age $(\mathrm{SD})$, years & $31.537(9.505)$ & $32.961(10.779)$ & $1.424(0.949)$ & 0.099 \\
\hline Female gender & $30.13(69 / 229)$ & $28.26(65 / 230)$ & $-0.019(0.043)$ & -0.029 \\
\hline Mean education (SD), years & $10.157(5.451)$ & $10.452(4.890)$ & $0.295(0.497)$ & 0.040 \\
\hline \multicolumn{5}{|l|}{ Type of education } \\
\hline Illiterate & $11.50(26 / 226)$ & $5.73(13 / 227)$ & $-0.058(0.026)$ & -0.146 \\
\hline Primary school & $40.71(92 / 226)$ & $44.93(102 / 227)$ & $0.042(0.047)$ & 0.060 \\
\hline High school & $27.88(63 / 226)$ & $27.75(63 / 227)$ & $-0.001(0.042)$ & -0.002 \\
\hline University & $19.91(45 / 226)$ & $20.70(47 / 227)$ & $0.008(0.038)$ & 0.014 \\
\hline Mean relatives (SD), $n$ & $1.655(2.413)$ & $1.409(1.978)$ & $-0.246(0.206)$ & -0.079 \\
\hline Mean children (SD), $n$ & $1.369(2.426)$ & $1.348(1.798)$ & $-0.021(0.200)$ & -0.007 \\
\hline \multicolumn{5}{|l|}{ Country of origin } \\
\hline Afghanistan & $14.41(33 / 229)$ & $14.35(33 / 230)$ & $-0.001(0.033)$ & -0.001 \\
\hline Iraq & $18.78(43 / 229)$ & $17.39(40 / 230)$ & $-0.014(0.036)$ & -0.025 \\
\hline Nigeria & $24.02(55 / 229)$ & $25.65(59 / 230)$ & $0.016(0.040)$ & 0.027 \\
\hline Pakistan & $9.61(22 / 229)$ & $8.26(19 / 230)$ & $-0.013(0.027)$ & -0.033 \\
\hline Syria & $28.38(65 / 229)$ & $28.26(65 / 230)$ & $-0.001(0.042)$ & -0.002 \\
\hline Other country & $4.80(11 / 229)$ & $6.09(14 / 230)$ & $0.013(0.021)$ & 0.040 \\
\hline \multicolumn{5}{|l|}{ Travel route } \\
\hline Balkan & $34.50(79 / 229)$ & $28.26(65 / 230)$ & $-0.062(0.043)$ & -0.095 \\
\hline Eastern & $19.65(45 / 229)$ & $22.17(51 / 230)$ & $0.025(0.038)$ & 0.044 \\
\hline African & $26.64(61 / 229)$ & $27.83(64 / 230)$ & $0.012(0.042)$ & 0.019 \\
\hline Other & $18.34(42 / 229)$ & $20.43(47 / 230)$ & $0.021(0.037)$ & 0.037 \\
\hline Detention during transition & $29.46(66 / 224)$ & $31.70(71 / 224)$ & $0.022(0.044)$ & 0.034 \\
\hline
\end{tabular}

came from Nigeria, and 18\% came from Iraq, followed by Afghanistan, Pakistan, and other bordering countries. The travel route for moving to Western Europe was through the Balkans for $28 \%$ of the participants in the $\mathrm{SH}+$ group and $34 \%$ of those in the ETAU group, followed by the African route, the Eastern route, and other travel routes. About $30 \%$ of the participants experienced detention during their transition to Europe. The 2 groups were similar with regard to most sociodemographic characteristics and baseline scores on outcomes, with the exception of the following variables: literacy, having distant relatives in the country of origin, detention duration, legal status conditions, and number of siblings. We added these variables in planned regression analyses accounting for imbalance between groups, without identifying relevant differences with respect to our main analyses on both primary and secondary outcomes (online suppl. Appendix, Table 4).

Assessment of more than $10 \%$ of $\mathrm{SH}+$ sessions showed near-perfect fidelity. We only identified small mistakes consisting of longer breaks (10-15 $\mathrm{min}$ ) in restarting the audio and taking more time for group discussion than allotted in the manual. Based on information collected at each site, the total supervision time required for all 5 sessions of an $\mathrm{SH}+$ group was $2 \mathrm{~h}$ on average per group.

Differences between study conditions on primary and secondary outcomes are reported in Table 1.

$\mathrm{SH}+$ led to a significant reduction in the frequency of any current mental disorders as measured with the MINI after the intervention (within 2 weeks from the last $\mathrm{SH}+$ session) (Cramer $V=0.135, p=0.012, \mathrm{RR}=0.499$; $95 \%$ CI 0.285-0.873), but not at the 6-month follow-up (primary outcome: Cramer $V=0.007, p=0.902, \mathrm{RR}=0.962$; $95 \%$ CI $0.521-1.778)$. The majority of detected mental disorders after the intervention were major depressive disorders ( 9 out of 171 participants in the $\mathrm{SH}+$ group and 20 out of 176 participants in the ETAU group), anxiety disorders (4 out of 171 participants in the $\mathrm{SH}+$ group and 7 out of 176 participants in the ETAU group), and PTSD (2 out of 171 participants in the SH+ group and 7 out of 176 participants in the ETAU group). At the 6-month follow-up, the majority of the detected mental disorders were major depressive disorders (12 out of 146 participants in the $\mathrm{SH}+$ group and 16 out of 157 participants in the ETAU group), anxiety disorders (2 out of 146 participants in the $\mathrm{SH}+$ group and 3 out of 157 participants 
Fig. 2. Trend in the frequency of any mental disorders over time.

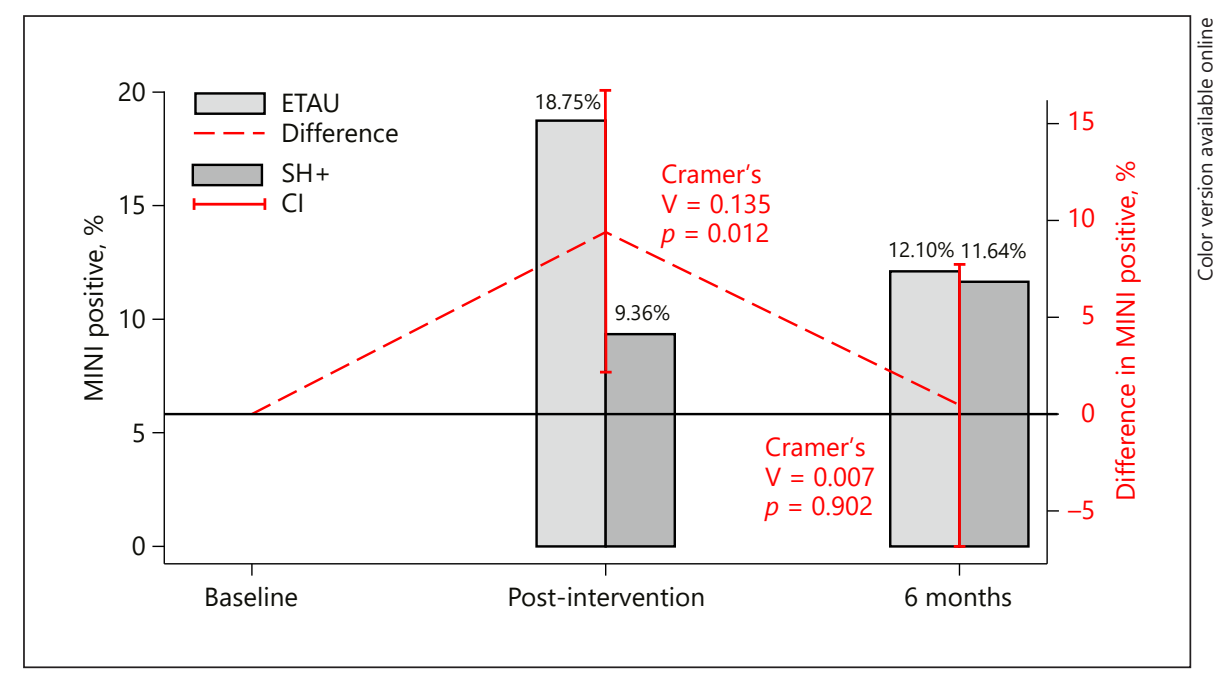

in the ETAU group), and PTSD (3 out of 146 participants in the $\mathrm{SH}+$ group and 3 out of 157 participants in the ETAU group). Figure 2 is a graphical representation of the trend over time in the frequency of current mental disorders in each of the 2 groups and of their difference.

$\mathrm{SH}+$, compared with ETAU, was also associated with larger improvements after the intervention for the secondary outcomes of psychological distress as measured with the GHQ-12 (standardized coefficient [Std Coef] = $-0.157, \mathrm{SE}=0.051, p=0.002)$, depression symptoms as measured with the PHQ-9 (Std Coef $=-0.125, \mathrm{SE}=0.050$, $p=0.013$ ), wellbeing as measured with the WHO-5 (Std Coef $=0.175, \mathrm{SE}=0.051, p=0.001$ ), and perceived problems as measured with the PSYCHLOPS (Std Coef = $-0.172, \mathrm{SE}=0.052, p=0.001)$. Such results were confirmed by the global statistical significance of the intervention on all outcomes by performing SURs $(p=0.001)$. At 6 months, we detected a statistically significant difference in favor of $\mathrm{SH}+$ on the WHO-5 (Std Coef $=0.187$, $\mathrm{SE}=0.050, p \leq 0.001$ ), while the other secondary outcome differences were not statistically significant. Results from SURs showed evidence of a global effect of treatment on secondary outcomes $(p=0.005)$ also at the 6-month follow-up. Results from the ANCOVA controlling for baseline scores were fully consistent with analyses using the LOCF approach (Table 1).

The ITT analysis results were confirmed in the PP analysis (online suppl. Appendix, Table 2). Secondary analyses of continuous outcomes conducted without any imputations of missing values did not identify any relevant difference with respect to the main analyses (online suppl. Appendix, Table 3).
We then proceeded to investigate the possible heterogeneity of the effect of treatment on outcomes by testing for interactions between intervention allocation and potential moderators. None of the interactions reached the statistical significance threshold for binary outcomes after application of the Bonferroni correction. By performing SURs on postintervention secondary outcomes, a global test on all interactions of the variable "intervention allocation," with center and the potential moderators on all regressions, turned out to be not statistically significant $(p=$ $0.581)$. The same test performed at the 6-month follow-up revealed interactions to be statistically significant ( $p=$ $0.025)$. We then conducted single regressions and found a global statistical significance of all interactions only in the regression having WHODAS as an outcome $(p<0.001)$. Thus, we performed simple models controlling for WHODAS at baseline and with the variable "intervention allocation", each separate variable, and their interaction as regressors. Only length of stay in the hosting country ( $p=$ $0.022)$ and years of education $(p=0.042)$ emerged as statistically significant moderators. As shown in online supplementary Appendix, Figure 1, a significant protective effect of SH+ on the WHODAS score at 6 months emerged for those who had already been in the host country for 6 years or longer. Post hoc analyses using the LOCF approach for the MINI, and considering deterioration of psychological symptoms as an additional outcome, did not show any differences between groups (online suppl. Appendix, Tables 5, 6). With regard to safety considerations, the ethics advisory board responded to 8 adverse events that were equally distributed between groups ( 5 in ETAU and 3 in $\mathrm{SH}+$ ), and none was evaluated to be related to the study/intervention participation. 


\section{Discussion}

This is the first study in refugees and asylum seekers resettled in Western Europe that examines the effects of a preventive intervention on the development of mental disorders. It is likely the largest intervention study ever conducted in this population group in high-income countries. Though we did not find a preventive effect of $\mathrm{SH}+$ on the presence of current mental disorders at the 6-month follow-up, which was our primary outcome, we found a significant difference in favor of $\mathrm{SH}+$ in reducing the frequency of current mental disorders immediately after the intervention.

Several reasons may explain the loss of effect over time, in particular the short duration of the intervention and a likely reduction of practice or application of the techniques after the group ended. A lack of participant blindness to the allocated interventions might be another reason, as knowledge of being allocated to the "enhanced intervention as usual" arm might have disappointed participants, leading to worse mental health conditions in the control group after the intervention. At 6 months, however, the control group participants could have moved beyond an initial disappointment, reversing to the same level of mental health problems as the intervention group [47]. However, analysis of secondary outcomes did not suggest any worsening in the control group mental health conditions, so a nocebo effect related to control group allocation seems unlikely. A third reason might be related to the nonspecific intervention ingredients of $\mathrm{SH}+$, which being transdiagnostic and based on the ACT might have generated a significant effect after the intervention, promoting flexibility and generic psychological skills that can be enhanced in any domain of life but not lasting over longer periods.

Interestingly, a decreased intervention effect over time was similarly observed in the $\mathrm{SH}+$ treatment trial conducted in Uganda, which involved a larger group of participants [18]. More generally, reviews on the effectiveness of psychosocial interventions in vulnerable populations have found that the beneficial effects of interventions on PTSD, depression, and anxiety symptoms were reduced at follow-up $[11,13]$. A recent systematic review of 17 RCTs involving 1,108 adult refugees confirmed a strong beneficial effect of psychological interventions in reducing PTSD and depression immediately after the intervention; this effect was reduced, but still statistically significant, at follow-up [8]. Compared to $\mathrm{SH}+$, these interventions generally provided a larger intervention "dose," for example because they were delivered as indi-

$\mathrm{SH}+$ for Preventing Mental Disorders in Refugees and Asylum Seekers vidual interventions or because the number of sessions was substantially greater. The length of follow-up was up to 6 months in the majority of the included studies [8]. In addition, there was a high heterogeneity across the included studies, indicating that there are moderators and mediators that need to be investigated in further analyses. Finally, meta-analyses from the available systematic reviews included very small studies, raising concerns regarding methodological standards and quality [48-50].

Little knowledge exists of whether these drops in effect sizes are due to intervention-related processes (e.g., a return to previous emotional/behavioral patterns), contextrelated variables (e.g., new or continued adversities experienced in the host countries, associated with renewed psychological distress), research-related variables (e.g., halo effect), or the impact that increased adversity may have on the effect of interventions over time. Likely, secondary analyses of the present study, and individual-participant data reanalyses of the whole body of randomized evidence on the efficacy of psychological interventions in vulnerable populations, will be able to ascertain which factors may contribute to maintenance versus loss of the effect over time.

For $\mathrm{SH}+$, it will be important to explore whether booster sessions after the delivery of the group intervention, administered face-to-face or through online devices [51], might assist in maintaining its benefits over time. Notably, booster sessions for $\mathrm{SH}+$ are possible, using the publication of the $\mathrm{SH}+$ illustrated book Doing What Matters in Times of Stress: An Illustrated Guide [25].

We note several limitations of our study. First, although this is the largest study in this population in highincome countries, we were only able to get close to, but did not reach, the planned sample size. Several potential factors may have contributed to the smaller sample size. As the included participants did not have a mental disorder, they may not have perceived their psychological wellbeing as a priority. Reported priorities included uncertainty about the refugee application, housing, unstable working conditions, and plans to move to another country. These factors may have limited engagement and availability to participate. Moreover, we identified a relatively large proportion of individuals with a diagnosis of mental disorder who were advised to seek appropriate care according to a predefined protocol and were excluded from participation. We note, however, that the systematic exclusion of participants with a mental disorder at baseline is a key strength of our study, as compared with previous studies evaluating psychological interventions on severely distressed participants who are more likely to 
respond to the intervention or to attend the sessions because they prioritize the intervention more $[52,53]$. These studies did not exclude participants with mental disorders at baseline, making it impossible to calculate the frequency of new cases of mental disorders at follow-up [54, 55]. Despite these challenges in reaching the target sample size, we were able to detect a statistically and clinically significant difference after treatment, thus indicating sufficient statistical power for this secondary analysis.

Second, a double-blind design was not feasible. However, outcome assessors were masked, i.e., they were not involved in any trial phase that might break their blindness $[56,57]$. For that reason, both participants and assessors were instructed not to mention any interventions received during the study.

A third limitation is related to the heterogeneity of the included population groups. The choice of involving people from multiple backgrounds was justified by the need to generate an evidence base for an intervention that has the potential for large-scale uptake across migrant populations.

Finally, a substantial proportion of participants was lost to follow-up (more than 20\% after the intervention and more than $30 \%$ at the 6 -month follow-up). We note, however, that the number of people lost to follow-up is in line with the expectations listed in the study protocol [19] and similar to those reported in other psychological intervention studies $[13,18]$.

Given the characteristics of SH+, i.e., delivery by briefly trained nonspecialist peers, its ease of implementation, the large group format, its short-term efficacy in preventing the development of mental disorders, its efficacy in ameliorating psychological distress in different populations, and the lack of adverse effects associated with its delivery, it might be considered as a public health strategy for healthcare agencies and nongovernmental organizations implementing migrant reception programs. Further research should be conducted to replicate results immediately after intervention and to explore the potential effects of booster $\mathrm{SH}+$ sessions to reinforce the beneficial effects even in the long term.

\section{Statement of Ethics}

This project was approved by the WHO Research Ethics Review Committee and by the ethics committees of all of the participating sites. Written informed consent was mandatory for all of the included participants. In accordance with the Declaration of Helsinki, the participants' confidentiality was preserved and the contents of the recruitment and follow-up forms were not disclosed to any third party.

\section{Conflict of Interest Statement}

The authors have no conflict of interests to declare.

\section{Funding Sources}

This work was supported by the European Commission (grant agreement No. 779255; "RE-DEFINE: Refugee Emergency: DEFining and Implementing Novel Evidence-based psychosocial interventions"). The authors are grateful to peer facilitators and cofacilitators, who delivered the $\mathrm{SH}+$ intervention, and to all of the assessors who administered baseline and follow-up interviews. The authors alone are responsible for the views expressed in this article, and they do not necessarily represent the views, decisions, or policies of the institutions with which they are affiliated.

\section{Author Contributions}

C.B. and M.v.O. conceived this study with inputs from K.C., M.P., and P.C. M.P. and C.B. drafted this paper. All the authors contributed to the study design, recruitment, follow-up assessments, and/or commenting on/editing the drafts of this work. F.T. planned and performed all of the statistical analyses with inputs from C.B.

The authors alone are responsible for the views expressed in this article and they do not necessarily represent the views, decisions, or policies of the institutions with which they are affiliated.

\section{Data Availability Statement}

Data collected in the RE-DEFINE study are stored in the online repository EUDAT B2SHARE (https://b2share.eudat.eu/records/ fa7264d624364683830ff37acee01c04). The reuse of data will be offered only upon motivated request, which will undergo the scrutiny of the RE-DEFINE General Assembly.

\section{References}

1 Turrini G, Purgato M, Ballette F, Nosè M, Ostuzzi G, Barbui C. Common mental disorders in asylum seekers and refugees: umbrella review of prevalence and intervention studies. Int J Ment Health Syst. 2017 Aug;11(1):51.

2 Jannesari S, Hatch S, Oram S. Seeking sanctuary: rethinking asylum and mental health. Epidemiol Psychiatr Sci. 2020 Aug;29:e154.

3 Sijbrandij M. Expanding the evidence: key priorities for research on mental health interventions for refugees in high-income countries. Epidemiol Psychiatr Sci. 2018 Apr;27(2):105-8.

4 Purgato M, Tol WA, Bass JK. An ecological model for refugee mental health: implications for research. Epidemiol Psychiatr Sci. 2017 Apr;26(2):139-41. 
5 Walther L, Kroger H, Tibubos AN, Tam Ta TM, von Scheve C, Schupp J, et al. Psychological distress among refugees in Germany: a cross-sectional analysis of individual and contextual risk factors and potential consequences for integration using a nationally representative survey. BMJ Open. 2020 Aug;10(8):e033658.

6 UNHCR. Emerging practices: mental health and psychosocial support in refugee operations during the COVID-19 pandemic [cited 2020 Oct 13]. Available from: https://www. unhcr.org/5ee2409b4/mental-health-andpsychosocial-support.

7 World Health Organization Regional Office for Europe. Interim guidance for refugee and migrant health in relation to COVID-19 in the WHO European Region [cited 2020 Oct 13]. Available from: https://www.euro.who. int/_data/assets/pdf_file/0008/434978/Interim-guidance-refugee-and-migranthealth-COVID-19.pdf.

8 Kip A, Priebe S, Holling H, Morina N. Psychological interventions for posttraumatic stress disorder and depression in refugees: $\mathrm{A}$ meta-analysis of randomized controlled trials. Clin Psychol Psychother. 2020 Jul;27(4):489-503.

9 Purgato M, Abdulmalik J, Prina E, Ceccarelli C, Tol WA, van Ginneken N, et al. Primarylevel and community worker interventions for the prevention of mental disorders and the promotion of wellbeing in low- and middleincome countries. Cochrane Database Syst Rev. 2021 Mar;2021(3):CD014722.

10 Uphoff E, Robertson L, Cabieses B, Villalón FJ, Purgato M, Churchill R, et al. An overview of systematic reviews on mental health promotion, prevention, and treatment of common mental disorders for refugees, asylum seekers, and internally displaced persons. Cochrane Database Syst Rev. 2020 Sep;2020(9):CD013458.

11 Nosè M, Ballette F, Bighelli I, Turrini G, Purgato $\mathrm{M}$, Tol W, et al. Psychosocial interventions for post-traumatic stress disorder in refugees and asylum seekers resettled in highincome countries: systematic review and meta-analysis. PLoS One. 2017 Feb;12(2):e0171030.

12 Turrini G, Purgato M, Acarturk C, Anttila M, Au T, Ballette F, et al. Efficacy and acceptability of psychosocial interventions in asylum seekers and refugees: systematic review and meta-analysis. Epidemiol Psychiatr Sci. 2019 Aug;28(4):376-88.

13 Purgato M, Gastaldon C, Papola D, van Ommeren M, Barbui C, Tol WA. Psychological therapies for the treatment of mental disorders in low- and middle-income countries affected by humanitarian crises. Cochrane Database Syst Rev. 2018 Jul;7(7):CD011849.

14 Purgato M, Gross AL, Betancourt T, Bolton P, Bonetto C, Gastaldon C, et al. Focused psychosocial interventions for children in lowresource humanitarian settings: a systematic review and individual participant data meta- analysis. Lancet Glob Health. 2018 Apr;6(4):e390-400.

15 Papola D, Purgato M, Gastaldon C, Bovo C, van Ommeren M, Barbui C, et al. Psychologi$\mathrm{cal}$ and social interventions for the prevention of mental disorders in people living in lowand middle-income countries affected by humanitarian crises. Cochrane Database Syst Rev. 2020 Sep;9(9):CD012417.

16 Barbui C, Purgato M, Abdulmalik J, Acarturk C, Eaton J, Gastaldon C, et al. Efficacy of psychosocial interventions for mental health outcomes in low-income and middle-income countries: an umbrella review. Lancet Psychiatry. $2020 \mathrm{Feb} ; 7(2): 162-72$.

17 Epping-Jordan JE, Harris R, Brown FL, Carswell K, Foley C, García-Moreno C, et al. SelfHelp Plus (SH+): a new WHO stress management package. World Psychiatry. 2016 Oct;15(3):295-6.

18 Tol WA, Leku MR, Lakin DP, Carswell K, Augustinavicius J, Adaku A, et al. Guided selfhelp to reduce psychological distress in South Sudanese female refugees in Uganda: a cluster randomised trial. Lancet Glob Health. 2020 Feb;8(2):e254-63.

19 Purgato M, Carswell K, Acarturk C, Au T, Akbai S, Anttila M, et al. Effectiveness and costeffectiveness of Self-Help Plus (SH+) for preventing mental disorders in refugees and asylum seekers in Europe and Turkey: study protocols for two randomised controlled trials. BMJ Open. 2019 May;9(5):e030259.

20 Castor EDC. Castor Electronic Data Capture [software]. Amsterdam; Castor EDC: 2017.

21 Kilic C, Rezaki M, Rezaki B, Kaplan I, Ozgen G, Sağduyu A, et al. General Health Questionnaire (GHQ12 \& GHQ28): psychometric properties and factor structure of the scales in a Turkish primary care sample. Soc Psychiatry Psychiatr Epidemiol. 1997 Aug;32(6):32731.

22 Sheehan DV, Lecrubier Y, Sheehan KH, Amorim P, Janavs J, Weiller E, et al. The Mini-International Neuropsychiatric Interview (M.I.N.I.): the development and validation of a structured diagnostic psychiatric interview for DSM-IV and ICD-10. J Clin Psychiatry. 1998;59 Suppl 20:22-33.

23 American Psychiatric Association. The Diagnostic and Statistical Manual of Mental Disorders. 5th ed. Washington: American Psychiatric Association; 2013.

24 World Health Organization. The ICD-10 classification of mental and behavioural disorders: clinical descriptions and diagnostic guidelines. Geneva: WHO; 1992.

25 World Health Organization. Doing what matters in times of stress: An illustrated guide. Geneva: WHO; 2020.

26 Hayes SC, Strosahl KD, Wilson KG. Acceptance and commitment therapy: An experiential approach to behavior change. New York: Guilford Press; 1999.

27 Spitzer RL, Williams Janet BW, Gibbon M, First MB. Structured Clinical Interview for DSM-III-R, Patient Edition/Non-Patient Edi- tion (SCID-P/SCID-NP). Washington: American Psychiatric Press; 1990.

28 World Health Organization. Catalogue of WHO psychiatric assessment instruments. Geneva: World Health Organization; 1995.

29 Blanchard EB, Jones-Alexander J, Buckley TC, Forneris CA. Psychometric properties of the PTSD Checklist (PCL). Behav Res Ther. 1996 Aug;34(8):669-73.

30 Weathers FW, Litz BT, Keane TM, Palmieri PA, Marx BP. Schnurr PP. The PTSD Checklist for DSM-5 (PCL-5): Standard [Measurement instrument] [cited 2020 Oct 13]. Available from: www.ptsd.va.gov.

31 Kroenke K, Spitzer RL, Williams JB. The PHQ-9: validity of a brief depression severity measure. J Gen Intern Med. 2001 Sep;16(9):606-13.

32 Ashworth M, Kordowicz M, Schofield P. The PSYCHLOPS (Psychological Outcome Profiles). Integr Sci Pract. 2012 Nov;2:36-9.

33 World Health Organization. Measuring health and disability: Manual for WHO Disability Assessment Schedule WHODAS 2.0. Geneva: WHO; 2010.

34 Heun R, Burkart M, Maier W, Bech P. Internal and external validity of the WHO WellBeing Scale in the elderly general population. Acta Psychiatr Scand. 1999 Mar;99(3):171-8.

35 EuroQol Group. EuroQol-a new facility for the measurement of health-related quality of life. Health Policy. 1990 Dec;16(3):199-208

36 Mollica RF, Caspi-Yavin Y, Bollini P, Truong T, Tor S, Lavelle J. The Harvard Trauma Questionnaire. Validating a cross-cultural instrument for measuring torture, trauma, and posttraumatic stress disorder in Indochinese refugees. J Nerv Ment Dis. 1992 Feb;180(2):111-6.

37 Riley A, Varner A, Ventevogel P, Taimur Hasan MM, Welton-Mitchell C. Daily stressors, trauma exposure, and mental health among stateless Rohingya refugees in Bangladesh. Transcult Psychiatry. 2017 Jun;54(3):304-31.

38 Normand ST, Landrum MB, Guadagnoli E, Ayanian JZ, Ryan TJ, Cleary PD, et al. Validating recommendations for coronary angiography following acute myocardial infarction in the elderly: a matched analysis using propensity scores. J Clin Epidemiol. 2001 Apr;54(4):387-98.

39 Austin PC. An Introduction to Propensity Score Methods for Reducing the Effects of Confounding in Observational Studies. Multivariate Behav Res. 2011 May;46(3):399-424.

40 Hemken D. Getting centered and standardized coefficients right. Stata Workshops [cited 2020 Oct 13]. Available from: http://www.ssc. wisc.edu/ hemken/Stataworkshops/stdBeta/ Getting\%20Standardized $\% 20$ Coeffi cients\%20Right.pdf.

41 Zellner A. An Efficient Method of Estimating Seemingly Unrelated Regressions and Tests for Aggregation Bias. J Am Stat Assoc. 1962 Jun;57(298):348-68. 
42 Baum CS, Schaffer M. Implementing econometric estimators with Mata. DC09 Stata Conference 1, Stata Users Group; 2009 JulSep.

43 Huisman JME. Item nonresponse: occurrence, causes, and imputation of missing answers to test items. Leiden: DSWO Press; 1999.

44 Millar P. HOTVALUE: Stata module to generate scales with missing values conditionally imputed. Statistical Software Components. 2008;(Nov):S456975.

45 Zhu C, Blizzard L, Stankovich J, Wills K, Hosmer DW. Be Wary of Using Poisson Regression to Estimate Risk and Relative Risk. Biostat Biom Open Access J. 2018 Feb;4(5).

46 StataCorp LLC. Stata Statistical Software: Release 16 [software]. College Station, TX; 2019.

47 Furukawa TA, Noma H, Caldwell DM, Honyashiki M, Shinohara K, Imai $\mathrm{H}$, et al. Waiting list may be a nocebo condition in psychotherapy trials: a contribution from network metaanalysis. Acta Psychiatr Scand. 2014 Sep;130(3):181-92.
48 Purgato M, Adams CE. Heterogeneity: the issue of apples, oranges and fruit pie. Epidemiol Psychiatr Sci. 2012 Mar;21(1):27-9.

49 Villar J, Carroli G, Belizán JM. Predictive ability of meta-analyses of randomised controlled trials. Lancet. 1995 Mar;345(8952):772-6.

50 IntHout J, Ioannidis JP, Borm GF, Goeman JJ. Small studies are more heterogeneous than large ones: a meta-meta-analysis. J Clin Epidemiol. 2015 Aug;68(8):860-9.

51 Wright JH, Caudill R. Remote Treatment Delivery in Response to the COVID-19 Pandemic. Psychother Psychosom. 2020;89(3):130-2.

52 Fournier JC, DeRubeis RJ, Hollon SD, Dimidjian S, Amsterdam JD, Shelton RC, et al. Antidepressant drug effects and depression severity: a patient-level meta-analysis. JAMA. 2010 Jan;303(1):47-53.

53 Buntrock C, Berking M, Smit F, Lehr D, Nobis $\mathrm{S}$, Riper $\mathrm{H}$, et al. Preventing Depression in Adults With Subthreshold Depression: Health-Economic Evaluation Alongside a Pragmatic Randomized Controlled Trial of a Web-Based Intervention. J Med Internet Res. 2017 Jan;19(1):e5.
54 van Zoonen K, Buntrock C, Ebert DD, Smit F, Reynolds CF 3rd, Beekman AT, et al. Preventing the onset of major depressive disorder: a meta-analytic review of psychological interventions. IntJEpidemiol.2014 Apr;43(2):318 29.

55 Cuijpers P, van Straten A, Warmerdam L, van Rooy MJ. Recruiting participants for interventions to prevent the onset of depressive disorders: possible ways to increase participation rates. BMC Health Serv Res. 2010 Jun;10(1):181.

56 Guidi J, Brakemeier EL, Bockting CL, Cosci F, Cuijpers P, Jarrett RB, et al. Methodological Recommendations for Trials of Psychological Interventions. Psychother Psychosom. 2018;87(5):276-84

57 Barbui C, Accordini S, Nosè M, Stroup S, Purgato $\mathrm{M}$, Girlanda F, et al.; CHAT (Clozapine Haloperidol Aripiprazole Trial) Study Group. Aripiprazole versus haloperidol in combination with clozapine for treatment-resistant schizophrenia in routine clinical care: a randomized, controlled trial. J Clin Psychopharmacol. 2011 Jun;31(3):266-73. 